\title{
CAUSAL LANGUAGE AND THE STRUCTURE OF FORCE IN NEWTON'S SYSTEM OF THE WORLD
}

\author{
Hylarie Kochiras
}

Although Newton carefully eschews questions about gravity's causal basis in the published Principia, the original version of his masterwork's third book contains some intriguing causal language. "These forces," he writes, "arise from the universal nature of matter." Such remarks seem to assert knowledge of gravity's cause, even that matter is capable of robust and distant action. Some commentators defend that interpretation of the text-a text whose proper interpretation is important since Newton's reasons for suppressing it strongly suggest that he continued to endorse its ideas. This article argues that the surface appearance of Newton's causal language is deceptive. What does Newton intend with his causal language if not a full causal hypothesis? His remarks actually indicate a way of considering the force mathematically, something he contrasts to the structure of the force as it really is in nature. In explaining that, he identifies a significant disjunction between the physical force itself and mathematical ways of considering it, and the text's significance lies in its view of the force's structure and in the questions raised about the relationship between mathematical representations and the physical world.

\section{Introduction}

Although Newton carefully eschews questions about gravity's causal basis in the published Principia, the original version of his masterwork's third book

Contact Hylarie Kochiras at Department of Philosophy and History of Science, National and Kapodistrian University of Athens, University Campus, Ano Ilisia 15771, Athens, Greece (hkochiras@ phs.uoa.gr).

This article underwent changes during my stays, first, as a European Union Institutes of Advanced Study fellow at New Europe College in Bucharest and, then, as a visiting scholar at the Department

HOPOS: The Journal of the International Society for the History of Philosophy of Science, vol. 3 (Fall 2013). 2152-5188/2013/0302-0002\$10.00. (C) 2013 by the International Society for the History of Philosophy of Science. All rights reserved. 
contains some intriguing causal language. That original version — suppressed by Newton and published only posthumously, in the original as De mundi systemate (1728a) and in translation as A Treatise of the System of the World (1728b) contains a lengthy description of the gravitational force that is quite unguarded. ${ }^{1}$ "These forces," he writes, "arise from the universal nature of matter already established." With these and some similar remarks, Newton appears to assert something that he later famously disavows: knowledge of gravity's cause. Indeed, he appears to be suggesting that matter is capable of unmediated distant action.

As the far more cautious tone of the published Principia reflects, even the appearance of such a suggestion would be unwelcome to many seventeenthcentury thinkers. For until Newton published his Principia in 1687, the mechanical philosophy in its Cartesian guise had seemed secure. ${ }^{3}$ The Cartesian understanding of mechanism accepted the principle that matter cannot act where it is not, taking motion to be communicable among bodies only by surface impacts, and that principle fit neatly with a plenum of matter since recourse to unmediated distant action is unnecessary if any two spatially separated bodies have continuous matter and thus a medium between them.

As Descartes understood this principle about mechanism and action, it supposed matter to be passive. Of course, interpretive questions may be raised about the extent of this passivity and about his associated principle of conservation of motion-that the universe's total quantity of motion always remains

of Philosophy and History of Science at the University of Athens. I gratefully acknowledge the support of both institutions and, in connection with the first, the financial contribution of the European Community's Seventh Framework Programme within the European Institutes for Advanced Study Programme. I would also like to thank Zvi Biener, Alan Nelson, and two anonymous referees for comments on an earlier draft, as well as the audiences at the HOPOS meeting in Budapest and at the University of Pittsburgh's Center for Philosophy of Science, where earlier versions of this article were presented.

1. The original version of the material that became the Principias third book was published posthumously, first in English translation, as noted, and then in Newton's original Latin under the same name given to the published Book III, De mundi systemate. As composed, the popularly styled text was the second of the two books into which Newton had divided the manuscript he gave Halley, De motu corporum, and seems to have been complete by November 1685 (Westfall 1980, 422). For further history of Book III's original version and its posthumous publication, see Westfall (1980, esp. 434 and 458-64), Cohen and Westfall in Newton (1995, 254), and Cohen (1999, 12-13). For a discussion of the manuscript known as De mundi systemate but associated with Newton's revisions for the Principiass third edition, see McGuire (1966).

2. "Oriri vero has vires ex universali natura materiae jam constitit" (Newton 1728a, 29). Translations are my own unless otherwise specified.

3. Elsewhere, I suggest a broad conception of mechanical philosophies, one that allows Newton's natural philosophy to qualify, in virtue of taking something from the simple machine tradition, in his case, the mathematical approach, and using it toward natural philosophy's traditional goal of identifying causes—-for Newton, forces. See Machamer et al. (2012). 
constant. Some interpretive questions concern the implications of Descartes's technical concept of motion, which is a numerical quantity, the product of size and speed. ${ }^{4}$ Others concern the principle's basis; Descartes grounds it in God's immutability, but scholars disagree about whether he ultimately takes matter to be passive in Malebranche's strong sense of denying efficient causation to everything except God. ${ }^{5}$ Such interpretive issues aside, however, on a surface reading Descartes allows matter a thin sense of activity, that of redirecting and redistributing existing motion. The universe's quantity of motion is "not something permanently fixed in given pieces of matter," he writes, "but something which is mutually transferred when collisions occur" (Descartes 1985, 243), but as a total quantity in the universe, it is fixed - motion being neither destroyed nor generated. On this reading, matter's capacity for action is considerably greater than that allowed by Malebranche but considerably less than that allowed by Renaissance vitalists and Scholastics. Whereas the Scholastics' substantial forms had allowed matter certain robustly active powers of generating new motion, as when a stone released from a height generates new motion by moving downward to seek the universe's center, Descartes construed such robustly active powers as mentalistic strivings and eliminated them from his reductive conception of matter. ${ }^{6}$

Scarcely a generation later, however, when Newton's 1687 Principia presented his gravitational theory, not only was the possibility of robustly active

4. Since Descartes understands motion as the product of size and speed, it is in principle possible that a body's quantity of motion could remain constant, even if that body generated new motion; this is because the body's size could be reduced so as to keep the product of size and speed constant. (I thank an anonymous referee for this point.) Yet the question of how a body's size could be reduced is not necessarily straightforward, given problems about how bodies are to be individuated in Descartes's plenum.

5. Certain remarks suggest what Daniel Garber has called the "cinematic view" (2001, 210), on which motion that appears to be transmitted among bodies is actually produced by God, as he recreates bodies, from one instant to the next, at different places; see in particular Principles of Philosophy (II.36; Descartes 1985, 240). A diachronic discussion of Descartes's ideas about body-body causation, preceded by a review of the extensive debate it, may be found in Machamer and McGuire 3(2009), chap. 3. As noted just below, however, my concern is the surface reading. I leave aside here questions about how God preserves motion in bodies and associated questions about whether Descartes is committed to occasionalism since, whatever position one takes in that debate, it remains true for Descartes that bodies themselves do not generate or destroy motion, and that is the point that Newton attacks in Query 31.

6. Descartes makes the point in connection with falling bodies: "What especially showed that the idea I had of heaviness was derived from that of mind was the fact that I thought that heaviness bore bodies toward the center of the earth as if it contained in itself some knowledge [cognitio] of it [i.e., the center of the earth]. For this could not happen without knowledge, and there cannot be any knowledge except in a mind" (AT VII 441-42; in Garber 1992, 98). 
matter back in play but so too was the possibility of unmediated distant action. ${ }^{7}$ Newton's language clearly indicated his belief that the gravitational force is causally efficacious; gravity keeps the moon in its orbit, he wrote. ${ }^{8}$ He also denied that there was any material medium between the earth and the moon, showing in Book II of the Principia that the heavens are nearly empty of matter. There cannot be a material medium pushing the planets along in their orbits since any medium dense enough to push them would also produce enough resistance to make them deviate from Kepler's idealized elliptical paths and eventually bring them to a stop (see Whiteside 1974, 6-8; Brackenridge 1995, 23). The clear implication of Newton's conclusions, his critics charged, was that he took bodies to be able to attract one another across a void, without any medium to convey the force. At the same time, Newton seemed to have turned the clock back to the time before Descartes had eliminated agency from matter. If gravitational effects were not produced by motion-transferring collisions in a material medium, perhaps bodies in the void could, all by themselves, generate motion where none had existed before. While Newton said nothing about Descartes's conservation principle in the Principia, he eventually attacked it directly, writing in one of the speculative essays appended to his Opticks, "It is very certain that there is not always the same quantity of motion in the world" (Query $31 ; 1952,397)$, and further speculating that since natural processes destroy motion, some motion-generating "active principles" must replenish the supply.

Granted, Newton emphasized throughout the Principia that his discoveries were confined to gravity's mathematical proportions, and he insisted in various texts, most famously in the 1713 General Scholium, that he made no claims as to gravity's cause. Still, his critics suspected him of being disingenuous, for where did active principles and the power to accelerate spatially distant bodies reside, if not in the bodies that he spoke of as attracting one another across a void? His critics thus suspected him of accepting that matter has robustly active powers of increasing the universe's scalar quantity of motion, and of doing so at a distance, without any spatially intervening medium.?

7. A classic discussion of Newton's senses of activity is in McMullin (1978a). McMullin refers to the robust sense of activity as "agent force in the full sense" and the "full, agent sense" $(41,82)$.

8. At Principia, Bk. III, Proposition 5, Scholium, Newton finally reveals that the centripetal force keeping planets in orbit is gravity $(1999,806)$ : "Hitherto we have called 'centripetal' that force by which celestial bodies are kept in their orbits. It is now established that this force is gravity, and therefore we shall call it gravity from now on. For the cause of the centripetal force by which the moon is kept in its orbit ought to be extended to all the planets, by rules 1, 2, and 4." See also Newton to the editor of the Memoirs of Literature (unpublished, London, ca. May 1712), in Newton (2004, 116): "For gravity without a miracle can keep the planets in."

9. It is worth nothing that the capacity to act robustly, such that the universe's scalar quantity of motion is increased, and the capacity to act distantly, such that spatially separated bodies can interact causally without any spatially intervening medium, are conceptually separable. Although Newton does 
Controversy about Newton's views remains possible today, despite the current availability of manuscripts unknown to his contemporaries, because the texts leave ample room for interpretive work. Yet there is one point on which Newton is steadfast: gravity is not essential to matter. ${ }^{10}$ The claim that gravity is not essential to matter therefore functions as a constraint on any interpretation of Newton, including one allowing robust and unmediated distant action. Superaddition is one such interpretation that respects the constraint. As construed by theologian Richard Bentley, who inquired after the theological implications of Newton's gravitational theory, gravity is an inessential attractive power; it does not belong to matter's essential nature but was superaddeddeliberately conferred by God. ${ }^{11}$ To attribute that view to Newton is to respect the constraint about essence by supposing that he fully detached the power of gravitational attraction from matter's nature. ${ }^{12}$

Another interpretation allowing robust and unmediated distant action is suggested, however, by the aforementioned passage in Newton's System of the World, and that is the one I consider here. My aim is not to determine whether

tend to associate the bearer of robustly active principles (whatever that may be) with the cause of forces acting between spatially separated bodies, there is no necessary connection between a capacity for robust action on the one hand and a capacity for unmediated distant action on the other.

10. See in particular Newton's concluding remarks to Rule 3: "I am by no means affirming that gravity is essential to bodies. By inherent force I mean only the force of inertia. This is immutable. Gravity is diminished as bodies recede from the earth" (1999, 795-96). See also his second letter to Bentley: "You sometimes speak of gravity as essential and inherent to matter. Pray do not ascribe that notion to me; for the cause of gravity is what I do not pretend to know, and therefore would take more time to consider of it" (Newton 2004, 100). Newton's reasons for denying that gravity is essential to matter may be diverse, including the recognition that the gravitational force would diminish to zero for infinitely separated bodies but perhaps also a desire to dissociate himself from any position suggesting the possibility of unmediated distant action. Newton also had occasion to correct Roger Cotes on this point, who in writing the preface to the Principia's second edition initially referred to gravity as an essential quality of matter. For a discussion of Cotes's probable reasons, see Biener and Smeenk (2012, sec. 3.3).

11. The view that Newton fully and consistently accepted action at a distance by attributing gravitational effects to active, inessential powers of matter superadded by God has been defended by John Henry $(1986,1994)$. The question of what superaddition amounts to has been debated in the secondary literature on Locke, and Locke, like Bentley but unlike Newton, does employ the term. Interpretations of Locke range from the deflationary "divine architect" view associated with Michael Ayers, that superadded qualities belong to the real essence and are simply those that God selected with special care, to the "glued on" or the "divine fiat" view associated with Margaret Wilson, that they do not belong to the real essence and were, so to speak, glued on by God after he created matter. The view of superaddition that Henry attributes to Newton resembles the "divine fiat" view.

12. Newton refers to the vis inertiae - the inherent force or force of inertia-as a quality that is inherent and essential to matter, as discussed in a subsequent section. What exactly he could mean by this is a point of controversy. On the one hand, he is consistent in the view that we have access only to appearances, not to the "essential and metaphysical constitution" of bodies or to innermost substances; the former remark occurs in the early text De gravitatione (Newton 2004, 27), and the latter, in the 1713 General Scholium (Newton 1999, 942), as well as in related drafts. On the other hand, in remarks 
Newton ever endorsed such a hypothesis, as I have addressed that question elsewhere. ${ }^{13} \mathrm{I}$ am rather interested in the meaning of this text's unguarded, apparently causal remarks about gravity. The text is an important one since its history suggests that Newton continued to endorse its ideas. Yet as I will argue, the surface appearance is deceptive. A careful analysis of Newton's reasoning reveals that he is not endorsing unmediated distant action there or indeed any full causal hypothesis at all. His remarks about causes actually denote a way of considering the force mathematically, something he contrasts to the structure of the force as it really is in nature.

When I speak of the structure of an entity, system, or process, I refer to its defining spatial properties and relations. ${ }^{14}$ For example, a sculpture's armature provides its spatial properties and thus its structure. More to the point here are the spatial relations between two gravitating bodies, both those along which the force is actually realized and those that are merely useful to employ. For in the System of the World, Newton strives to eliminate the (epistemic) possibility that the gravitational force between two bodies consists in two distinct unidirectional attractions, a possibility raised by the mathematical ways of considering the force. In doing so, he implies a notable disjunction between mathematical ways of considering the force and the physical force itself. The significance of the passage, therefore, does not lie in an answer to the question about gravity's full cause, since it provides none. The significance rather lies in Newton's claims about the force's structure and in the questions those claims raise about the relationship he saw between mathematical ways of considering features of the world and those physical features themselves.

\section{Causal Language in Newton's System of the World}

In Book III of the Principia, Newton applies the mathematical principles developed in the earlier books to the physical world, showing that the force known to us from terrestrial phenomena as gravity extends to all matter throughout the universe, keeping the moon and the planets in their orbits. As noted earlier,

following Rule 3 he does distinguish between a quality that is essential or inherent, as the vis inertiae is, and one that is merely universal, as gravity is (795). I will not engage the debate here but will proceed under the assumption that he sees some genuine distinction between a universal quality and an essential one, even if we are able to speak only of some Lockean nominal essence.

13. I have argued elsewhere that while Newton entertains various hypotheses about gravity's cause, including unmediated distant action by robustly active matter, he never endorsed any of them (see Kochiras 2009, 2011).

14. On structural explanations in Newton and his contemporaries, see Alan Gabbey (1985). Gabbey has argued that Newton may be understood as providing structural explanations in the sense introduced in McMullin (1978b). 
however, the work ultimately published as Book III, which employs the same technical style as the preceding books, replaces a version written "in popular form, so that it might be more widely read," which Newton suppressed. As he explains in remarks prefatory to the published version, he did so in order "to avoid lengthy disputations" by excluding mathematically benighted readers, who might be unable to lay aside their "preconceptions." ${ }^{15}$ Although the System of the World does differ in some substantive ways from the version that supplanted it (it includes, e.g., a justification of the Copernican-Keplerian model that he later decided was unnecessary; Westfall 1980, 434), we have little reason to suppose that Newton meant to repudiate it; his stated reason for suppressing it suggests no such thing, and the substance of the two versions is largely continuous.

Again, however, the System of the World contains some very unguarded remarks. The early passages contain no hint of anything unusual, as Newton circumscribes his intentions in ways familiar from the Principia itself-from its full title, Mathematical Principles of Natural Philosophy, and from its text. In Principia Book I, for instance, when he elaborates on his definition of the motive quantity of the centripetal force, Newton cautions his readers that this concept is "purely mathematical" and signifies nothing about the force's "physical causes and sites" (Definition VIII; Newton 1999, 407). He echoes that remark (and presages his much later General Scholium announcement that he does not pretend to know gravity's cause and feigns no hypotheses) in the System of the World. His purpose is to determine the force's quantity and properties and to investigate its effects "mathematically"; indeed, he writes, by employing the general name "centripetal force," he can avoid determining its appearance "by hypothesis." 16

15. Newton's extended introductory comments are as follows: "In the preceding books I have presented principles of philosophy that are not, however, philosophical but strictly mathematical. . . . It still remains for us to exhibit the system of the world from these same principles. On this subject, I composed an earlier version of book 3 in popular form, so that it might be more widely read. But those who have not sufficiently grasped the principles set down here will certainly not perceive the force of the conclusions, nor will they lay aside the preconceptions to which they have become accustomed over many years; and therefore, to avoid lengthy disputations, I have translated the substance of the earlier version into propositions in a mathematical style, so that they may be read only by those who have first mastered the principles" (1999, 793).

16. "For us, the purpose is to discover the quantity and properties themselves, and to investigate the effect in moving bodies mathematically; and so that we do not determine the appearance of it [i.e., the force] by hypothesis, we call that which tends somehow toward a center by the general name 'centripetal'" (Nobis propositum est quantitatem \& proprietates ipsius eruere, atque effectus in corporibus movendis investigate mathematice: proinde ne speciem ejus hypothetice determinemus, diximus ipsam generali nomine centripetam, quae tendit in centrum aliquod; Newton 1728a, 3). 
Newton's distinction between mathematical and physical considerations of physical systems and forces will be relevant to my discussion in section 4. As a methodological tool critical to the results he achieved, it has drawn a good deal of attention. I. B. Cohen, for instance, has explored in detail what he calls the "Newtonian Style," in which Newton assigns all considerations not directly pertinent to mathematical physics, including questions about physical causes, to later stages of investigation. ${ }^{17}$ More recently, Andrew Janiak has explicated Newton's distinction by emphasizing that, for Newton, treating the gravitational force mathematically does not mean regarding the law as merely a calculating device (as suggested by his expositor Samuel Clarke) but rather as showing that force, a physical quantity, can be measured, via measurements of other quantities, namely, the masses and accelerations of a system's component bodies. ${ }^{18}$ It is this latter understanding that will be relevant in section 4 .

Having stated his intention to consider the force only mathematically, Newton sets out to show that gravitation is universal. ${ }^{19}$ Yet while that surely remains the central aim of the System of the World, just as it is the central aim of its published successor, Newton abandons his declared restriction midway through his reasoning, and writes as follows.

Since, however, the action of the centripetal force upon an attracted body be proportional, at equal distances, to the matter in this body, it is fitting to reason that it be proportional to the matter in the attracting body. Indeed, the action is mutual, and makes the bodies mutually approach one another by a mutual endeavor (by the third law of motion), and hence it must be the same in each body. It can be so considered, one body as attracting, the other as attracted; but this distinction is more mathematical than natural. The attraction is really of each body upon the other, and is exactly the same kind in each.

And hence the attractive force is found in both. The sun attracts Jupiter and the other planets; Jupiter draws its satellites; and by the same reason-

17. Cohen discusses the Newtonian Style in multiple places (1980, chap. 3, 52-53, esp. 60-64; also 132; see also Cohen 1999, 60-61).

18. Clarke's exposition may be found in Alexander (1956), 5.110-16, 5.118-23. Passages in which Newton treats the force his law identifies as causally efficacious include Principia, Bk. III, Proposition 5, Scholium (Newton 1999, 806). Janiak explicates the distinction between mathematical and physical treatments of force in the context of showing how Newton could be justified in claiming to have discovered the causally efficacious gravitational force itself, identified by his law, even as he concedes that he has not discovered gravity's physical basis. For the details of illuminating arguments, see of Janiak (2008), chap. 3, esp. 60-64.

19. Important discussions of Newton's argument for universal gravitation include McMullin (1978a, 62-69), McGuire (1995, 278), Cohen (1999, esp. 19), and William Harper (2002, 174-201). 
ing, the satellites mutually act upon one another and upon Jupiter; and all the planets mutually upon one another. And though the mutual actions of a pair of planets can be distinguished from one another, and considered as two actions, by which each draws the other, still insofar as they are intermediate, they are not two, but one single action between two endpoints. By the contraction of a single, interceding rope, two bodies can be drawn toward one another. The cause of the action is twofold, indeed the disposition of each body; the action likewise is twofold, to the extent that it is upon two bodies: but to the extent that it is between two bodies, it is single and unitary. It is not that the sun draws Jupiter by one action and that Jupiter draws the sun by another; but by one action, the sun and Jupiter endeavor to approach one another. By the action that the sun draws Jupiter, Jupiter and the sun mutually endeavor to approach one another (by the third law of motion), and by the action that Jupiter draws the sun, Jupiter and the sun mutually endeavor to approach one another. The sun, however, is not drawn to Jupiter by a twofold action, nor Jupiter to the sun by a twofold action, but it is one intermediate action by which both mutually approach one another. ${ }^{20}$

Despite Newton's original stated intent of considering the force's effects mathematically, here he contrasts a mathematical way of considering the force with the force as it is in nature, focusing his attention on the latter. ${ }^{21}$

20. "Cum autem actio vis centripetae in corpus attractum, paribus distantiis, proportionalis sit materiae in hoc corpore; rationi etiam consentaneum est, ut sit proportionalis materiae in corpore trahente. Etenim actio mutua est, facitque corpora conatu mutuo (per Motus Legem 3) accedere ad invicem, \& proinde sibi ipsi conformis esse debet in corpore utroque. Considerari potest corpus unum ut attrahens, alterum ut attractum, sed haec distinctio magis mathematica est quam naturalis. Attractio revera est corporis utriusque in utrumque, atque adeo ejusdem generis in utroque.

"Et hinc est quod vis attractiva reperiatur in utroque. Sol trahit Jovem \& caeteros Planetas, Jupiter trahit Satellites; \& paritate rationis, Satellites agunt in se invicem \& in Jovem, \& Planetae omnes in se mutuo. Et quamvis binorum Planetarum actiones in se mutuo distingui possint ab invicem, \&, ut actiones binae, quibus uterque trahit alterum, considerari: tamen quatenus intermediae sunt, non sunt binae, sed operatio simplex inter binos terminos. Contractione funiculi unius intercedentis possunt bina corpora ad invicem trahi. Causa actionis gemina est, nimirum dispositio utriusque corporis; actio item gemina quatenus in bina corpora: at quatenus inter bina corpora simplex est \& unica. Non est una operatio qua Sol v. g. trahit Jovem, \& alia operatio qua Jupiter trahit Solem, sed una operatio qua Sol \& Jupiter conantur ad invicem accedere. Actione qua Sol trahit Jovem, conantur Jupiter \& Sol ad se mutuo accedere per Motus Leg. 3) \&, actione qua Jupiter trahit Solem, conantur etiam Jupiter \& Sol ad se mutuo accedere: Sol autem non attrahitur actione duplici in Jovem, neque Jupiter actione duplici in Solem, sed una est actio intermedia qua ambo accedunt ad se mutuo" (Newton 1728a, 24-26).

21. I will discuss the structure of the force in a later section. See also Stein's discussion of the System of the World passages; in connection with these and other texts, Stein argues that Newton introduces a new concept of a natural power or force of nature as (i) having the form of a "central field of force" and 
Newton seems to stray even further from his original intent by speaking of gravity in causal terms; in the second paragraph just quoted, he writes, "The cause of the action is twofold, indeed the disposition of each body." Some subsequent passages contain similar remarks: "In this manner we conceive a single action, exercised between two planets and arising from the conspiring natures of each." ${ }^{22}$ And further on, in explaining the attractions among the parts of a planet, Newton writes, "In truth these forces arise from the universal nature of matter already established, and on account of them the forces of the component particles produce the force of the entire sphere."23

\section{A Full Causal Interpretation and Its Problems}

These remarks, with their striking causal language, certainly command attention. For what could Newton possibly intend by such remarks, if not a full causal hypothesis? Since he points to the "dispositions" in the bodies as the "twofold" cause of the force, and further says that the force arises from the "universal nature of matter," perhaps he intends 'disposition' in the sense of 'power', and perhaps he means to suggest the following full causal hypothesis: ${ }^{24}$ matter has the power of attracting other material particles, and this power belongs to the very nature or essence of each material particle, yet that power is distinct from the gravitational force itself, which is relational, and can arise only in a universe containing at least two particles (noted in more detail below). In a one-particle universe, matter's essential power to attract would not be expressed, and the force could not arise. This causal hypothesis would pose no conflict to the aforementioned constraint, that is, Newton's consistent position that gravity itself is not essential to matter. For again, the force itself is relational; indeed, it is an enormously complex relation. ${ }^{25}$ Thus, even if the gravitational

(ii) being characterized by a "unity of interaction." Every force of nature is identified by its law, with the vis inertiae, the one passive force, which is not a central field force, being identified by all three laws of motion (see Stein 2002, 282-91).

22. Emphasis added. The original reads as follows: "Ad hunc modum concipe simplicem exerceri inter binos Planetas ab utriusque conspirante natura oriundam operationem" (Newton 1728a, 26).

23. Emphasis added. "Oriri vero has vires ex universali natura materiae jam constitit, \& propterea ex particularum viribus componi vim globi totius” (Newton 1728a, 29).

24. Like Stein $(2002,188)$ and Motte (presumably the author of the 1728 translation; 1728b, 39), I have translated Newton's dispositio as 'disposition'. Since the force depends on the distance between the planets, not an individual planet's position in absolute space, the context seems to rule out 'position' or 'placement'. Nevertheless, it is by no means clear that Newton's dispositio should be understood as a power.

25. Commentators remarking on the gravitational force's relational character refer to the "actual," as opposed to "dispositional," sense in which Newton uses the term, to follow McMullin's terminology. (When McMullin denies that Newton ever asserts gravity to be relational, he is referring to gravity as a 
force arose from powers that were essential to matter, as a relation, the force itself could not belong to any single particle, and thus, as a one-particle universe indicates, it could not belong to a material particle's essence. ${ }^{26}$

Thus, if Newton did mean to assert the positive claim of the causal hypothesis just sketched-that matter essentially possesses a power to attract that is causally efficacious toward producing the gravitational force-that claim would be consistent with the constraint about essence. If he further asserted the negative claim that no medium plays any role in producing or conveying the force, that would amount to an interpretation that respects the constraint but that nevertheless grounds the force in something essential to matter, namely, the power to attract.

Was the preconception that Newton feared many readers would have been unable to lay aside, then, the principle that matter cannot act where it is not? Was the reason that he ultimately withheld the System of the World perhaps that some of its passages advance a full causal hypothesis about gravity, one supposing matter to be robustly active and capable of unmediated distant action, such that there is nothing more to the physical story?27 A number of commen-

"disposition or capacity"; see McMullin 1978a, $142 \mathrm{nn} .114$ and 119; see also 59-60, as referenced in my n. 26 below). On the relational aspect of the gravitational force, see also Jammer (1957, 90-91), Weinert (2004), and Schliesser (2011).

26. On this point, see McMullin, who identifies Newton's various uses of the term 'gravity' and notes that a particle alone in a void would have gravity in the sense of being disposed to attract and be attracted but not in the sense of actually doing so (1978a, 59-60). Schliesser, by way of arguing that Newton accepts unmediated distant action in the System of the World, also notes that the gravitational force itself does not belong to a single particle $(2011,80)$. That Newton's System of the World does not take a single particle to give rise to the gravitational force is one point on which Schliesser and I agree.

27. As Newton's remarks here are spare and rather enigmatic, we cannot know for certain which "disputations" he wished to avoid. One possibility pinpoints Hooke's charge that Newton stole from him the knowledge of the inverse-square force and his concomitant demand for recognition. The force was suspected to have the inverse-square proportions, and while a question posed by Hooke provided impetus for Newton's insight, it was Newton alone who had derived the force mathematically (on this point, see Westfall 1980, 387). He meant to stake his exclusive claim to it by underscoring the discovery's mathematical requirements — and to accomplish that by producing a version too mathematically demanding for Hooke to understand fully. This account retains adherents, including George Smith. (See Smith 2007, sec. 3: "When Hooke raised a priority issue on inverse-square forces, Newton dropped the original version of the last book, switching to presenting the system of the world in a sequence of mathematically argued propositions, many of which demand far more of the reader than anything in the original version.") Yet the account has also been questioned; Westfall argues that Newton made many of the changes to Book III before his dispute with Hooke and that the dispute was a result of Newton's state of tension and the excitement of discovery, as he rapidly reworked Book III (1980, 459-60). Another possibility (compatible with the first) is that Newton hoped to avoid or at least reduce the criticism he was likely to receive about his claim of universal gravitation or about the possibility that his theory implied of action at a distance. Eric Schliesser defends that latter possibility, arguing that the System of the World presents Newton presents a causal hypothesis that embraces distant action as physically real and that the disputes he means to avoid concern philosophical prejudices 
tators have thought so. Although the published Principia eschewed the question about gravity's "physical cause," writes Richard Westfall, "when he first wrote his discovery down, he was less reserved; the forces proportional to the quantity of matter, he said, 'arise from the universal nature of matter'" $(1980,422)$. In a commentary coauthored with Westfall, I. B. Cohen agrees, and a version of the interpretation just sketched has recently been defended by Eric Schliesser. ${ }^{28}$

Despite its causal language, however, the System of the World does not in fact advance either the positive or the negative claim required for the full causal hypothesis just sketched. ${ }^{29}$ To consider the negative claim first, at no point in the text does Newton deny the existence of a medium. In fact, at one point he blithely assumes the existence of an aether. In concluding his discussion of aggregate bodies, he states that the parts of the other planets mutually attract, just as the parts of the earth do, causing all of these bodies to have spherical shapes; their parts cohere, he continues, "and are not diffused through the aether." ${ }^{30}$

against distant action: "The prejudices he has in mind are not merely 'vulgar,' but rather philosophical, that is, those in circulation amongst the learned (especially, Hooke and Huygens). For the Treatise is more speculatively metaphysical than the published version of Book III" (Schliesser 2011, 81).

28. Although Cohen and Westfall do not provide details, and in fact mention only God and the aethers as specific causal hypotheses that Newton considers, they seem to imply some version of the hypothesis sketched here when writing, "Newton's young followers in the early eighteenth century returned to the assertion of the System of the World that universal gravitation arises from the nature of matter" (Cohen and Westfall in Newton 1995, 256). Schliesser (2011) develops and defends the interpretation in detail, as mentioned in n. 26 above.

29. Although my arguments about activity were briefly previewed in one paragraph of a previous article (Kochiras 2011, 181-82), that article focused mainly on contextualizing and further explaining the epistemological "substance counting problem" that I identified earlier (in Kochiras 2009) and defending the view from critics, including Schliesser. Thus, that response to Schliesser focused primarily on explaining the following items. (i) The substance counting problem cannot be dissolved by distinguishing the one true substance, God, from created immaterial substances (see Kochiras 2011, 17071). (ii) I quite agree that Newton's rational mechanics includes the methodological resources to avoid the substance counting problem. My purpose was not to say otherwise but rather to understand why Newton sometimes pursues speculations that push the boundaries of empiricism and to understand the implications of those speculations (see 172). (iii) I take Newton's epistemic attitude to be merely that of inclination, not commitment, as my critics erroneously charged, and I allow that he did sometimes consider the possibility of unmediated distant action (see Kochiras 2009, 275; 2011, 172-73). This current article closely follows Newton's reasoning in System of the World, to understand what his causal language means, if not any full causal hypothesis; thus, while sec. 3 elaborates the points about activity briefly previewed earlier and rejects such interpretations of the causal language that Schliesser gives, sec. 4 provides an alternative interpretation.

30. "Utque partes eorum cohaereant \& non spargantur per aethera" (Newton 1728a, 29). The negative claim is attributed to Newton by Schliesser, who claims that Newton remains "completely silent on the possibility of a medium of transmission" and thereby tacitly indicates his belief that none exists (Schliesser 2011, 86). As indicated, however, Newton is not completely silent and in fact allows the existence of an aether in the quoted remark. One might argue, of course, that 'aether' does not refer here to a medium but rather to an extramundane void or the regions of space beyond the clouds- which was a common seventeenth-century meaning, as an anonymous referee for this journal notes. Yet Newton does 
This last remark tells against the negative claim, suggesting that questions about whether a medium exists and about gravity's cause are tangential to his intent in this text.

As for the positive claim, the main reason for denying that the System of the World advances it is one just suggested: Newton's intent in the passage turns out to be something else entirely. I shall develop my arguments for that in the next section, however. For the moment, I want to cast some initial doubt on the notion that he is advancing the positive claim here by noting how poorly it fits with texts that explicitly mention gravity's cause and matter's nature. In Query 31, a late text with earlier antecedents, which was added for the 1717-18 edition of the Opticks, Newton publicly speculates about gravity's cause: "Seeing therefore the variety of motion which we find in the world is always decreasing, there is a necessity of conserving and recruiting it by active principles, such as are the cause of gravity" $(1952,399)$. These active principles (whose full nature and location remain indeterminate) are active in a very robust sense, a sense that evokes Aristotle's belief that, without some generative source, motion would eventually cease, and the universe would reach stasis.

That robust sense of activity differs considerably from the thin sense of activity that Newton takes to belong to matter's nature in virtue of its characteristic quality, the vis inertiae. As Definition 3 of the Principia indicates, a body's vis inertiae — or more accurately, its exerted vis inertiae — enables a body to deliver the reaction required by Law 3; it enables it to change the state of a body impressing a force on it by reciprocating with an impressed force ${ }^{31}$ For as a number of commentators have noted, an exertion of the vis inertiae seems to amount to an impressed force. ${ }^{32}$ Now, if gravitational attractions involve new

frequently use the term to refer to a medium. One text temporally proximate to the System of the World is the published Principia, and there Newton writes of "the action of aether or of air or of any medium whatsoever-whether corporeal or incorporeal” (Principia, Bk. I, Sec. 11, Scholium; Newton 1999, 561). Early texts in which an aether is a medium include Newton's letter to Boyle; the printed, unpublished sheets discussed by Cohen (1958; see esp. 372); and Newton's December 7, 1675, letter to Oldenburg (in Newton 1959-71, 1:364-65). Later texts include the Opticks (see, e.g., Newton 1952, 364-65 and 368).

31. As Newton writes in Definition 3 of the Principia, "Inherent force may also be called by the very significant name of force of inertia. Moreover, a body exerts this force only during a change of its state, caused by another force impressed upon it, and this exercise of force is, depending on the viewpoint, both resistance and impetus: resistance insofar as the body, in order to maintain its state, strives against the impressed force, and impetus insofar as the same body, yielding only with difficulty to the force of a resisting obstacle, endeavors to change the state of that obstacle" (1999, 404-5).

32. In connection with this point, see Howard Stein and Alan Gabbey. Stein writes: "Whereas the 'force of inactivity' is a permanent attribute of a body—not always exercised, but always presentimpressed force is by its nature episodic. The explanation ends with the remark, "Impressed forces are of different origins; as from percussion, from pressure, from centripetal force" $(2002,284)$. As Alan 
motions, then a reciprocating impressed force must involve the robust sense of activity, and yet as Newton conceives it, the vis inertiae could not be the source of such a robust action. ${ }^{33}$ In and of itself, the vis inertiae provides for only a thin sort of activity, one facilitating only the redirection and redistribution of existing motion. That is Newton's point in Query 31 when he calls the vis inertiae a "passive principle." While it is not passive in Malebranche's full sense, the vis inertiae facilitates only the redirection or redistribution of existing motion, and so "by this principle alone, there never could have been any motion in the world" (Newton 1952, 397-99).

If Newton identified the sense of activity belonging to matter's nature via its vis inertiae with the robust sense that Query 31 cites as gravity's cause, he would be grounding gravity's cause in the essence of matter. ${ }^{34}$ But instead of identifying the two, Newton distinguishes them and attributes only the vis inertiae's thin sense of activity to matter's nature, ${ }^{35}$ while citing only the robust active

Gabbey reads Newton, (i) Newton does think that "a vis inertiae can at the same time be a vis impressa," and (ii) accordingly, the exercised vis inertiae is not only proportional to body, "it is also proportional to induced change of state" $(1980,275)$. Gabbey identifies two reasons that "Newton did not make the proportionality between 'exercised' vis inertiae and change of state clear in his explanatory paragraph following the definition of the force" (275; see the same page for the two reasons Gabbey discusses). There are difficulties, of course, with the view that Newton took the exerted or exercised vis inertiae to constitute an impressed force. It would, e.g., require that we suppose Newton's remark "This force is always proportional to the body" to be incomplete, for the vis inertiae would be proportional not only to the body (the quantity of matter) but also to acceleration. The need for this supposition was pointed out to me by Andrew Janiak.

33. See McMullin's discussion of this point: "The duality of active and passive principles is muted in the Principia, as we have seen, but it is by no means absent. From where does new motion come in this new system of the world? In his account of vis inertiae, Newton appears to attribute to the 'impulse' elicited by way of opposition to impressed force a surprisingly active role in affecting the state of motion of the agent body. Yet vis inertiae could not be the ultimate source of material agency. There is a world of difference between 'action' and 'reaction,' between the attraction the sun, for example, exerts on the earth (when considered as attraction, i.e., as rooted in some 'active principle' initiating motion) and the reaction of the earth on the sun (considered as reaction, i.e., as rooted in the vis inertiae of the earth)" (1978a, 45).

34. The claim that Newton employs a single sense of activity both in Definition 3 and in Query 31 has been suggested recently by Eric Schliesser, who defends a version of the interpretation developed in this section. In connection with his identification of what I have delineated as two distinct senses of activity, see Schliesser (2011), 85, 90, 91-92, and 97. Some authors simply fail to distinguish the two senses carefully (see, e.g., Machamer et al. 2012, sec. 1).

35. Although Query 31 opens with the suggestion that "the small Particles of Bodies [have] certain Powers, Virtues, or Forces, by which they act at a distance," Newton appears to be speculating that gravity is not the only force acting between spatially separated bodies, but without committing himself to any claims about the physical causes of those forces. For the suggestion is soon followed by this caveat: "How these attractions may be performed, I do not here consider. What I call attraction may be performed by impulse, or by some other means unknown to me. I use that word here to signify only in general any force by which bodies tend towards one another, whatsoever be the cause" (1952, 375-76). Elsewhere in Query 31, Newton seems reluctant to locate active principles in matter; he writes that 
principles as gravity's cause. ${ }^{36}$ All of this turns out to be compatible with Newton's System of the World, for gravity's full, physical cause is not his concern in that text, as we will see in the next section.

\section{A Structural Interpretation}

As we have seen, the System of the World contains a qualitative description of gravity, one whose causal language, when considered in isolation, seems to suggest a full causal hypothesis. Yet if we follow Newton's reasoning through the passages that precede and follow that description, it reveals a different intent.

The main thread of reasoning begins as Newton explains the proportion between force and bodies. His point is that for attracted bodies at equal distances from some central attracting body, the centripetal force is proportional to the bodies' masses alone, not to anything else. Although there is only one proportion at issue, he speaks as if there were two, dividing the discussion into two parts. He does so because he is concerned not only to state that the force is proportional to mass but also to show that (for equal distances) it is not proportional to anything else. Thus, the two parts of the discussion differ in the feature that Newton finds to be irrelevant to the force. The irrelevant feature that he attends to first is the bodies' magnitude. "That bodies of such diverse magnitudes so nearly agree in the proportion of their forces is certainly not without mystery," he writes, but such is his discovery. "The weights of all the planets are to one another as the forces," and the force is independent of the bodies' sizes. ${ }^{38}$ The other irrelevant feature is composition ("modes," as he writes in a later passage).$^{39}$ For equal distances, the force is proportional to mass

matter is "moved by" active principles, a locution that leaves the location of active principles unspecified. This reluctance is much more pronounced in a draft related to Query 31 in which Newton writes that the nature of the active principle and its relation to matter is "difficult to explain"; although he crosses out that thought, he does so without attempting to explicate the relationship. He also retains the suggestion that matter is passive. This draft, University Library, Cambridge, Add. 3970, fol. 620r, is discussed by McGuire $(1968,170-71)$. Another classic discussion of Newton's struggle to understand active principles may be found in McMullin (1978a). My own discussion of Newton's uncertainty may be found in Kochiras (2011), sec. 6.4.

36. It remains possible, of course, that Newton nevertheless attributed some quite distinct power of robust activity to matter, yet as noted earlier, I have addressed that question elsewhere (see Kochiras 2011), and my aim here is understand his remarks in the System of the World.

37. "Corpora magnitudinis tam diversae ad analogiam cum viribus tam prope accedere, mysterio certe non caret" (Newton 1728a, 21).

38. "Ea ratione constabit optime pondera Planetarum omnium esse inter se ut vires" (Newton 1728a, 22).

39. "Vires autem quae sunt ut materia in omnium formarum corporibus terrestribus, atque adeo non mutantur cum formis, reperiri debent in corporibus universis, tam coelestibus, quam terrestribus; 
alone and is independent of the bodies' composition. In the case of celestial bodies, he explains, it must be true that for equal distances, mass alone determines the force's magnitude, for to say otherwise conflicts with observations; if composition played a role, the bodies would not be retracted from the tangents of their orbits in the proportions that have been found. ${ }^{40}$ Similarly, in the case of bodies near the surface of the earth, mass alone is relevant, Newton's experiments having shown that pendulum bobs of equal mass "descend in equal times through equal spaces," regardless of whether they are made of gold, wood, water, or wheat (Newton 1728a, 23-24).

Having narrowed the quantities determinative of the gravitational force to mass and distance, Newton now draws out the consequences to reach the claim of universal gravitation. He explicitly denies that the circumsolar force, as he here calls it, draws a planet to the sun and that the circumterrestrial force draws a stone to earth, without any symmetric action taking place. ${ }^{41}$ That is to say, he denies that the force is asymmetrical. It cannot be that the earth is solely an attracting body, which exerts the force, while the moon or the stone is solely an attracted body, on which the force is exerted, he explains. There cannot be any distinction among massive bodies such that some only exert the force, without being acted on by it, while others are only acted on, without exerting the force. This is a consequence of the proportionality of the force to mass, as expressed in this earlier-quoted remark: "Since, however, the action of the centripetal force upon an attracted body be proportional, at equal distances, to the matter in this body, it is fitting to reason that it be proportional to the matter in the attracting body." Gravitation is universal in scope, then, with every body being both an attracting and an attracted body - both source and subject of the force. ${ }^{42}$ At this point, he begins to contrast the way we can consider the force and the gravitating bodies to the way they actually are. To consider the earth, for instance, as an attracting body and the stone as an attracted body is "more mathematical than natural." For mathematical purposes, we might consider a given body solely as an attracted body or solely as an attracting body, but no such distinction can be

$\&$ in omnibus esse proportionalies materiae: eo quod haec omnia, non genere substantiae, sed formis \& modificationisbus solummodo, differunt" (Newton 1728a, 28).

40. It is also required by reason, Newton explains, that the same proportionality holds at the level of the planet's constituent particles, for if the force varied not only with mass but also composition at the level of the constituent particles, then since those particles are indeed the constituents of the compound body (the planet), the force on the planet would vary with its composition as well as its mass.

41. In these passages, Newton speaks generally of the "centripetal force" and also of the "circumsolar," "circumterrestrial," and "circumjovial" forces, in part because he has not yet emphasized the identity of these forces and in part because he employs such terms when he is speaking mathematically, considering the force in terms of a specific central body of interest.

42. Cohen discusses this point (1999, 18); see again Stein's discussion (2002, esp. 288). 


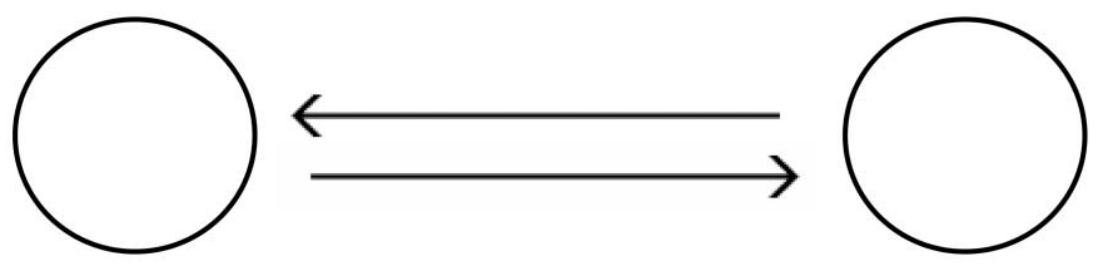

Figure 1. Attraction as two distinct unidirectional forces

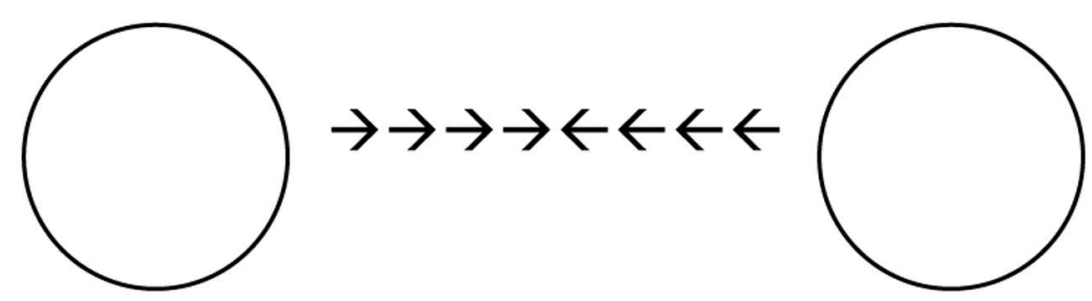

Figure 2. Attraction as a single mutual endeavor

drawn if we consider the force as it really is in nature: "The attractive force is found in both. ... And by the same reasoning, the satellites mutually act upon one another and upon Jupiter; and all the planets mutually upon one another."

The point that Newton has defended so far is that the force cannot be asymmetric in the sense that one body is only the force's source while another body is only its subject, and if he sought to claim only that gravity is universal, he might close his case here. However, he is intent on showing something more, something about the force's actual structure: the attraction between two bodies does not consist in two distinct unidirectional forces emitted by each on the other (fig. 1) but is rather a single mutual endeavor (fig. 2).

This is the point Newton is making in the above-quoted passages containing the curious causal language noted earlier. Although "the cause of the action is twofold; indeed the disposition of each body," it is not a "twofold action" by which the sun and Jupiter attract one another but "one intermediate action." ${ }^{43}$

43. There are several senses of 'gravity' and 'action' implicit in Newton's discussion. In connection with other texts, McMullin makes such distinctions explicit (1978a, sec. 3.2): "The gravity of a body can be construed as either active (A) or as passive (P), depending on whether the body is considered as attracting or being acted upon. It can be actual (E) or dispositional (D), depending on whether gravity is attributed to a body on the basis of its actually exercising attracting (or actually being attracted) or only on the basis of its capacity to act (or be acted upon). Thus, for instance, a body alone in a void would not exhibit gravity in the former sense, but would in the latter. Finally, 'gravity' could refer either to the weight (W) or to the mass (M) of a body." 
As he explains further, "The action is mutual, and makes the bodies mutually approach one another by a mutual endeavor. ... And though the mutual actions of a pair of planets can be distinguished from one another, and considered as two actions, by which each draws the other, still insofar as they are intermediate, they are not two, but one single action between two endpoints." Newton's concern in this passage, then, is to eliminate the epistemic possibility that for any pair of attracting bodies - say, the earth and a stone or the earth and its moon-each is the source of a distinct unidirectional force on the other, such as we might draw in a vectorial representation.

With this purpose in mind, let us reconsider the remark mentioning a twofold cause: "The cause of the action is twofold, indeed the disposition of each body." Although taken in isolation, this remark suggests the full causal hypothesis' positive claim about gravity, in context we can notice that it belongs to Newton's explanation of how the force may be considered mathematically. To see this, it is helpful to recall the long passage quoted earlier, in section 2, and to notice its first sentence, which ends with the claim that the force "be proportional to the matter in the attracting body." Here, in connection with establishing gravity's universality, Newton asserts the proportionality of its magnitude to quantity of matter, thereby considering the force mathematically.

He devotes the rest of the passage to explaining the structure of force as it is in nature, contrasting that against the ways that the force may be considered mathematically. He states that contrast explicitly. In a system of two gravitating bodies, we might consider one body only as attracted, which is to say we might selectively attend to the effect on it; however, he explains, "this distinction is more mathematical than natural." The remainder of the passage reiterates and elaborates that contrast. In doing so, Newton first provides a vivid visual analogy. We should not think that each of two gravitating bodies acts independently on the other, as if each grasped the other by means of its own rope. It is rather that the two planets approach one another by means of a single rope contracting between them.

With that visual image in place, Newton reiterates that the force can be considered mathematically; now mentioning two ways of doing so-by considering the mass that each body contributes to the force and by considering the acceleration that each undergoes— he again emphasizes that this differs from the force's actual structure. This is the sentence employing causal language. ("The cause of the action is twofold, indeed the disposition of each body; the action likewise is twofold, to the extent that it is upon two bodies: but to the extent that it is between two bodies, it is single and unitary.") In speaking of a twofold cause, Newton neither makes mention of the robust "active principles" that preoccupy him elsewhere nor gives any other indication of concern with 
the question about gravity's physical cause. In noting the twofold cause, he is simply restating the point that he made at the passage's outset, namely, that the force's magnitude is proportional to the quantity of matter, and again, as it concerns only quantities, this is a mathematical way of considering the force. The sentence thus notes two ways of considering the force mathematically: selectively focusing on each body's mass and selectively focusing on each body's acceleration, the latter representable by the unidirectional arrows that fail to represent the force's actual structure as a mutual endeavor.

While none of this rules out the possibility that Newton nevertheless did attribute to matter some robust power of generating new motion, there is nothing in this text to suggest that possibility. The sentence containing the causal language and indeed the entire passage aims to explain the force's actual structure, as distinct from the way it might be considered mathematically.

At this point, however, another question arises. If Newton's purpose is, as just argued, to explain the nature of the gravitational force itself, as contrasted with mathematical ways of considering it, what explains his confidence that the force has the singular structure that he describes? The disjunction between the way of considering the force mathematically, as if it consisted in two unidirectional endeavors, and the singular structure that Newton asserts the force actually to have in nature does not appear in the Principia, the discussion to which it belongs not having been carried over into the published Principia. Indeed, it seems at odds with his stance in the Principia. ${ }^{44}$ In various places, notably his author's preface to the Principia, Newton takes mathematical findings to denote real features of the physical world. The preface expresses the general claim that mathematical principles can be fruitfully applied to philosophy's basic problem, which is "to discover the forces of nature from the phenomena of motions." ${ }^{45}$ The disjunction evident in the System of the World does not, of course, concern the force's proportions as an inverse-square force or, accordingly, his claim to have discovered the force. Yet he does express confidence that its actual structure differs from the vectorial representation noted, and we might ask after the source of that confidence. I see only a partial explanation,

44. For a recent discussion of a range of questions and difficulties concerning Newton's attempts to confer certainty on natural philosophy via mathematics, see Guicciardini (2009), esp. 21, 325.

45. Newton (1999), 382. Other remarks in the preface indicate that Newton there identifies mathematical principles with mechanical ones and then takes those mechanical/mathematical principles to be the means of identifying forces. (It is because "we are concerned with natural philosophy ... gravity, levity, elastic forces, resistance of fluids, and forces of this sort" that the Principia "sets forth mathematical principles of natural philosophy"; that Newton identifies these mathematical principles with mechanical ones is evident because his subsequent remark assigns the same task of discovering forces to 'mechanical principles': "If only we could derive the other phenomena of nature from mechanical principles by the same kind of reasoning!"; 382). 
but perhaps that is not too surprising; explaining the connection between mathematical findings and the natural world is a challenge, even in the seventeenthcentury context, which was marked by an increasing confidence that mathematical findings had realist implications (Henry 2002, chap. 3, esp. 14-15).

When he first asserts the mutual nature of the force, at the outset of the passage, Newton cites his third law, yet it is difficult to see how that law could adjudicate between a force consisting in two unidirectional endeavors and one consisting in a single mutual endeavor. (And although his interest in the force's actual structure follows after his claim that gravitation is universal, it does not seem motivated by it since universal gravitation is consistent with the mathematically useful structure as well as the actual one.) In a later passage, however, Newton repeats his claim about the force's singular structure, this time in connection with the behavior of iron filings near a lodestone, and that discussion reflects the Principiąs recommendation for investigating forces. The first step for investigating forces, he explains in the Principia, is to determine the quantities and proportions that "follow from any conditions that may be supposed"; once that is done, the physical species, causes, and proportions of the forces may be investigated, by "coming down to physics" and comparing the proportions found to be mathematically necessary "with the phenomena." 46 An influential predecessor was Isaac Barrow, whose lectures Newton heard, and as Antoni Malet has shown, Barrow's view implied testing to be the means of determining which mathematically true and hence physically possible world is the "physically true" world (Malet 1997, 278-79). ${ }^{47}$ This approach is reflected in the System of the World's discussion of the lodestone.

Iron draws the lodestone equally as the lodestone draws the iron; for all iron in the vicinity of the iron also draws other iron. But the action between the lodestone and the iron is single, and is considered as single by the philosophers: the operation of the iron upon the lodestone is really the operation of the lodestone between itself and the iron, as both endeavor mutually to approach one another. This is evident, for with the removal of the lodestone the force of all the iron nearly ceases.

46. Principia, Scholium to Bk. I, Sec. 11 (Newton 1999, 588-89).

47. Newton himself recalled Barrow as probably influential on his ideas about generating geometrical figures kinematically: “tis probable that D' Barrows Lectures might put me upon considering the generation of figures by motion, tho I not now remember it" (Geometrical Lectures, translated from the Latin edition, revised, corrected, and amended by Edmund Stone [London, 1735], in Garrison [1987], 614). Commentators who have identified and discussed Barrow's influence include Garrison (1987), De Gandt (1995, 209), Malet (1997), and Guicciardini (2009). Guicciardini investigates Newton's ideas about geometry's method and its relation to natural philosophy in considerable depth. 
In this manner we conceive a single action, exercised between two planets and arising from the conspiring natures of each. And this action continues in the same way to each; as it is proportional to the matter appearing in one of them, it will be proportional to the matter in the other. ${ }^{48}$

Here Newton points to observations of bodies subject to another centripetal force, magnetism. He draws a conclusion about the structure of the magnetic force in the first paragraph, and then in the second paragraph, he applies the conclusion to gravity. If the magnetic attraction between the lodestone and the piece of iron consisted in two distinct unidirectional forces, each exerted by the one body on the other, then the removal of the lodestone should remove only the force exerted by the lodestone on the iron, Newton reasons, leaving the force exerted by the iron intact-in which case the effect on the neighboring bits of iron would remain. Instead, the effect on those neighboring bits of iron is (nearly) eliminated with the removal of the lodestone. That result does lend some credence to the claim that the force between the piece of iron and the lodestone is a single mutual endeavor. Yet it seems only to illustrate, not to justify, Newton's belief that the gravitational force also has that singular structure, and in fact Newton's final remark in the passage suggests that he intends only to illustrate. One cannot help wondering whether ideas of mutual sympathies, familiar from alchemical texts, might have motivated his belief that the force's actual structure is singular and mutual, and there is an intriguing resemblance to the "magnetic coition" figuring in William Gilbert's de Magnete, yet even if that could be shown, it would show influence, not justification. ${ }^{49}$

In regard to my earlier conclusion about Newton's causal language, his discussion of the lodestone provides further support. The purposes he reveals in this and other cases underscore the extent to which the question about gravity's full causal story lies outside his current concerns. The point he is making by means of the lodestone and the magnetic force has nothing to do with causes; he does not mention any powers in the bodies and says nothing about

48. "Ferrum trahit magnetem aeque ac Magnes ferrum; nam ferrum omne in vicinia Magnetis trahit etiam aliud ferrum. At actio inter Magnetem \& ferrum simplex est, \& a Philosophis consideratur ut simplex: Operatio ferri in Magnetem ipsa est Magnetis operatio seipsum inter \& ferrum, qua ambo conantur accedere ad se mutuo. Id ex eo manifestum est, quod sublato magnete cessat prope vis tota ferri.

"Ad hunc modum concipe simplicem exerceri inter binos Planetas ab utriusque conspirante natura oriundam operationem; \& haec eodem modo se habebit ad utrumque: adeo proportionalis existens materiae in uno eorum, proportionalis erit materiae in altero" (Newton 1728a, 26).

49. On Gilbert's distinction between coition, which is mutual, and attraction, which is not mutual, see Stephen Gaukroger (2006), 364. 
a medium, either to endorse or exclude the possibility of its existence. His purpose in considering the magnetic force is simply to bolster his claim about the gravitational force's structure as a "single action" between two planets. His remark about the planets' "conspiring natures," so curious when considered out of context, directly follows his attempt to show that the magnetic force is a single action, and when we consider it in its context in the passage above, we see that it pertains to the conclusion about the force's actual structure.

As a further indication that Newton is concerned solely with matters connected to gravitation's universality and structure, his next move is to rebut an objection he anticipates will be made against the thesis of universal gravitation. ${ }^{50} \mathrm{He}$ then denies that the thesis is disproved by the fact that we do not observe small bodies such as stones attracting either one another or the earth. ${ }^{51}$ The force is simply too small to be perceived. Toward the end of this series of passages, he emphasizes that for all bodies, whether terrestrial or celestial, the force is proportional to the quantities of matter and does not vary with the body's form or modes..$^{52}$ And since the forces "arise from the universal nature of matter," the force of any aggregate body is made up of the force of its parts. Here again, however provocative that phrase is when considered both out of context and in connection with the controversy about distant action, considering it in context shows that Newton is not connecting it to the causal question but only to the thesis of universal gravitation.

50. The arguments may be found in Newton (1728a), 26-28.

51. The objection that Newton here anticipates together with his rebuttal are recast in the published Principia as Book III's Proposition 7, Corollary 3: "Therefore the gravity toward the whole planet arises from and is compounded of the gravity toward the individual parts. We have examples of this in magnetic and electric attractions. For every attraction toward a whole arises from the attractions toward the individual parts. This will be understood in the case of gravity by thinking of several smaller planets coming together into one globe and composing a larger planet. For the force of the whole will have to arise from the forces of the component parts. If anyone objects that by this law all bodies on our earth would have to gravitate toward one another, even though gravity of this kind is by no means detected by our senses, my answer is that gravity toward these bodies is far smaller than what our senses could detect, since such gravity is to the gravity toward the whole earth as [the quantity of matter in each of] these bodies to the [quantity of matter in the] whole earth" (Newton 1999, 811).

52. "The forces, which are as the matter in terrestrial bodies of all forms, and therefore are not mutable with the forms, must be found in all sorts of bodies whatsoever, celestial as well as terrestrial, and be in all proportional to their quantities of matter, because among all there is no difference of substance, but of modes and forms only. But in the celestial bodies, the same thing is likewise proved thus. We have shewed, that the action of the circumsolar force upon all the planets (reduced to equal distances) is as the matter of the planets; That the action of the circumjovial force upon the satellites of Jupiter observes the same law; and the same thing is to be said of the attraction of all the planets towards every planet. But thence it follows that their attractive forces are as their several quantities of matter" (Newton 1728a, 43-44). 


\section{Conclusion}

Newton's System of the World contains a strikingly unguarded description of the gravitational force, and since it is likely, given the text's history, that he continued to endorse it, the description is important to understand. As we have seen, some commentators have interpreted it as advancing a full causal hypothesis, and Newton's remark that "the cause of the action is twofold" initially seemed to intimate that he supposes matter capable of robust and unmediated distant action. Yet neither of the claims needed for the interpretation found support. Instead of advancing the negative claim that no medium is required to produce or convey the force, he showed no interest in questions about a medium, even casually assuming the existence of an aether as he attended to other questions. As for the positive claim that matter essentially possesses a robustly active disposition to attract, one causally efficacious toward producing the gravitational force, that drew no support from other texts; the interpretation depended on an identity between two senses of activity that those other texts clearly distinguish. More to the point, however, the remarks in the System of the World most suggestive of a full causal hypothesis turned out to mean something quite different from what first appearances suggested. In particular, the force's "twofold" cause of action turned out to refer to quantities, specifically quantities of matter, and thus also to a way of considering the force mathematically. In mentioning those ways of considering the force, Newton's purpose in mentioning those ways of considering the force was to present them as contrast cases, against which the force's actual structure, as it is in nature, might be understood. Throughout the text, we saw, his attention remained focused on questions about the scope of gravitation and the structure of the force. Since he claims the force's actual structure to differ from mathematical ways of considering it, a further question arose concerning the basis of his confidence about that. That question, of course, connects to broader ones about the relationship he saw between the physical world itself and mathematical ways of considering it. And those difficult questions persist, the narrower question about justification being only partly answered by the Princip$i d$ s recommended investigatory procedure, that of "coming down to physics" and observation, once the mathematical proportions have been discovered.

\section{REFERENCES}

Alexander, H. G., ed. 1956. The Leibniz-Clarke Correspondence. Manchester: Manchester University Press. 
Biener, Z., and C. Smeenk. 2012. "Cotes' Queries: Newton's Empiricism and Conceptions of Matter." In Interpreting Newton, ed. Andrew Janiak and Eric Schliesser, 105-37. Cambridge: Cambridge University Press.

Brackenridge, Bruce. 1995. The Key to Newton's Dynamics: The Kepler Problem and the Principia. Berkeley: University of California Press.

Cohen, I. Bernard. 1958. "Versions of Isaac Newton's First Published Paper." Archives Internationales d'Histoire des Sciences 11:357-75.

1980. The Newtonian Revolution. Cambridge: Cambridge University Press. . 1999. "A Guide to Newton's Principia." In Newton 1999, 1-370.

De Gandt, Francois. 1995. Force and Geometry in Newton's "Principia." Trans. Curtis Wilson. Princeton, NJ: Princeton University Press.

Descartes, Rene. 1985. The Philosophical Writings of Descartes. Vols. 1 and 2. Trans. John Cottingham, Robert Stoothoff, and Dugald Murdoch. Cambridge: Cambridge University Press.

Gabbey, Alan. 1980. "Force and Inertia in the Seventeenth Century: Descartes and Newton." In Descartes: Philosophy, Mathematics, and Physics, ed. S. Gaukroger, 270-72. Sussex: Harvester.

. 1985. "The Mechanical Philosophy and Its Problems: Mechanical Explanations, Impenetrability, and Perpetual Motion." In Change and Progress in Modern Science, ed. Joseph Pitt, 9-84. Dordrecht: Reidel.

Garber, Daniel. 1992. Descartes' Metaphysical Physics. Chicago: University of Chicago Press. . 2001. Descartes Embodied. Cambridge: Cambridge University Press.

Garrison, James W. 1987. "Newton and the Relation of Mathematics to Natural Philosophy." Journal of the History of Ideas 48 (4): 609-27.

Gaukroger, Stephen. 2006. The Emergence of a Scientific Culture: Science and the Shaping of Modernity, 1210-1685. Oxford: Oxford University Press.

Guicciardini, Niccolo. 2009. Isaac Newton on Mathematical Certainty and Method. Cambridge, MA: MIT Press.

Harper, William. 2002. "Newton's Argument for Universal Gravitation." In A Cambridge Companion to Newton, ed. I. Bernard Cohen and George E. Smith, 174-201. Cambridge: Cambridge University Press.

Henry, John. 1986. "Occult Qualities and the Experimental Philosophy: Active Principles in Pre-Newtonian Matter Theory." History of Science 24:335-81.

- 1994. "'Pray Do Not Ascribe That Notion to Me': God and Newton's Gravity.” In The Books of Nature and Scripture: Recent Essays on Natural Philosophy, Theology and Biblical Criticism in the Netherlands of Spinoza's Time and the British Isles of Newton's Time, ed. James E. Force and Richard H. Popkin, 123-47. Dordrecht: Kluwer.

- 2002. The Scientific Revolution and the Origins of Modern Science. 2nd ed. Hong Kong: St. Martin's.

Jammer, Max. 1957. Concepts of Force. Cambridge, MA: Harvard University Press. Janiak, Andrew. 2008. Newton as Philosopher. Cambridge: Cambridge University Press.

Kochiras, Hylarie. 2009. "Gravity and Newton's Substance Counting Problem." Studies in History and Philosophy of Science 40:267-80. 
. 2011. "Gravity's Cause and Substance Counting: Contextualizing the Problems." Studies in History and Philosophy of Science 42:167-84.

Machamer, Peter, and J. E. McGuire. 2009. Descartes's Changing Mind. Princeton, NJ: Princeton University Press.

Machamer, Peter, J. E. McGuire, and Hylarie Kochiras. 2012. "Newton and the Mechanical Philosophy: Gravitation as the Balance of the Heavens." Southern Journal of Philosophy 50:370-88.

Malet, Antoni. 1997. "Isaac Barrow on the Mathematization of Nature: Theological Voluntarism and the Rise of Geometrical Optics." Journal of the History of Ideas 58:265-87.

McGuire, J. E. 1966. "Body and Void and Newton's De Mundi Systemate: Some New Sources." Archive for History of Exact Sciences 3:206-48.

. 1968. "Force, Active Principles, and Newton's Invisible Realm." Ambix 15:154-208.

-. 1995. "Transmutation and Immutability: Newton's Doctrine of Physical Qualities." Repr. in Tradition and Innovation: Newton's Metaphysics of Nature, ed. J. E. McGuire, 262-86. Boston: Kluwer.

McMullin, Ernan. 1978a. Newton on Matter and Activity. Notre Dame, IN: University of Notre Dame Press.

. 1978b. "Structural Explanation." American Philosophical Quarterly 15 (April): 139-47.

Newton, Isaac. 1728a. De mundi systemate: Liber Isaaci Newtoni. London: Tonson, Osborn, $\&$ Longman.

- 1728b. A Treatise of the System of the World. Trans. [Andrew Motte?]. London: Fayram.

- 1952. Opticks; Or, A Treatise of the Reflections, Refractions, Inflections and Colors of Light. Based on the 4th ed. of 1730. New York: Dover.

- 1959-71. Correspondence of Isaac Newton. Ed. H. W. Turnbull, J. F. Scott, A. R. Hall, and L. Tilling. 7 vols. Cambridge: Cambridge University Press. . 1995. Newton: Texts, Backgrounds, Commentaries. Ed. I. B. Cohen and R. S. Westfall. New York: Norton.

. 1999. The Principia: Mathematical Principles of Natural Philosophy. Trans. I. Bernard Cohen and Anne Whitman. Berkeley: University of California Press.

. 2004. Newton: Philosophical Writings. Ed. Andrew Janiak. Cambridge: Cambridge University Press.

Schliesser, Eric. 2011. "Without God: Gravity as a Relational Quality of Matter in Newton's Treatise." In Vanishing Matter and the Laws of Motion: Descartes and Beyond, ed. Dana Jalobeanu and Peter Anstey, 80-100. London: Routledge.

Smith, George. 2007. "Newton's Philosophiae Naturalis Principia Mathematica." In Stanford Encyclopedia of Philosophy, ed. Edward N. Zalta. Stanford, CA: Stanford University. http://plato.stanford.edu/entries/newton-principia/.

Stein, Howard. 2002. "Newton's Metaphysics." In A Cambridge Companion to Newton, ed. I. Bernard Cohen and George E. Smith, 256-307. Cambridge: Cambridge University Press.

Weinert, Friedel. 2004. The Scientist as Philosopher: Philosophical Consequences of Great Scientific Discoveries. Dordrecht: Springer. 
Westfall, Richard S. 1980. Never at Rest: A Biography of Isaac Newton. New York: Cambridge University Press.

Whiteside, Derek Thomas, ed. 1974. The Mathematical Papers of Isaac Newton. Vol. 6, 1684-1691. Cambridge: Cambridge University Press. 